\title{
An evaluation of existent methods for estimation of embankment dam breach parameters
}

\begin{abstract}
The study of dam-break analysis is considered important to predict the peak discharge during dam failure. This is essential to assess economic, social and environmental impacts downstream and to prepare the emergency response plan. Dam breach parameters such as breach width, breach height and breach formation time are the key variables to estimate the peak discharge during dam break. This study presents the evaluation of existing methods for estimation of dam breach parameters. Since all of these methods adopt regression analysis, uncertainty analysis of these methods becomes necessary to assess their performance. Uncertainty was performed using the data of more than 140 case studies of past recorded failures of dams, collected from different sources in the literature. The accuracy of the existing methods was tested, and the values of mean absolute relative error were found to be ranging from 0.39 to 1.05 for dam breach width estimation and from 0.6 to 0.8 for dam failure time estimation. In this study, artificial neural network (ANN) was recommended as an alternate method for estimation of dam breach parameters. The ANN method is proposed due to its accurate prediction when it was applied to similar other cases in water resources.
\end{abstract}

Keyword: Embankment dam; Dam breach; Breach parameters; Uncertainty analysis 